\title{
The Dunlop test and reading in primary school children
}

\author{
J F STEIN, P M RIDDELL, AND M S FOWLER \\ From the University Laboratory of Physiology, Oxford and Royal Berks Hospital, Reading.
}

SUMmaRY Using the Dunlop synoptophore test we have examined the reliability of vergence control for small fusion targets in 753 primary school children aged 7-11, and we have compared these results with the reading performance of 451 of them. $30 \%$ of the total sample of children had unstable responses in the Dunlop test. The proportion decreased with age, ranging from $49 \%$ of 5 -year-olds to only $11 \%$ of 10 -year-olds. The reading of children who had developed accurate vergence control was on average 6.3 months in advance of those who had not. Those with unstable Dunlop test responses were much more likely to be backward or low normal readers than children with stable responses. We conclude that in experienced hands the Dunlop test is a useful indicator of the development of vergence control and that immaturity of vergence control may contribute to children's reading problems.

We have recently put forward the hypothesis ${ }^{1}$ that many children with specific reading difficulties (developmental dyslexics) may suffer a disorder of binocular vergence control when they inspect small fusional stimuli. Their poor vergence control may lead them to mislocate, missequence, and misorientate letters when they try to read small print. We have been using the Dunlop synoptophore test of vergence stability ${ }^{2}$ to investigate such children with reading difficulties. In 1982 we reported that between half and two-thirds of dyslexic children had abnormal responses in this test, ${ }^{3}$ whereas very few normal readers suffered these problems. However, Newman et $a l .{ }^{4}$ have recently found in a sample of 298 unselected primary school children that there were almost as many normal as backward readers who gave abnormal responses in the Dunlop test. Their high proportion of 'false positives' casts doubt on the reliability of this test for helping with the management of children with reading problems.

Over the past few years we also have been examining a large number of unselected primary school children in order to determine the incidence of abnormal responses in the Dunlop test in a 'normal' population. We obtained reading age information about these children after the orthoptic examinations

Correspondence to $\mathrm{Dr} J$ F Stein, University Laboratory of Physiology, Parks Road, Oxford OX13PT. had been completed, so that, like Newman et al., we could compare the Dunlop test results with reading performance. However, we found fewer false positives than Newman et al., and we confirmed that many more backward than normal readers have abnormal responses in the Dunlop test. Preliminary reports of some of these findings have already been published. ${ }^{57}$

\section{Material and methods}

\section{SUBJECTS}

We visited 11 primary schools in widely different neighbourhoods around Reading which had kindly agreed to participate in these studies. In all, 753 children aged between 5 and 11 were examined. Each child was given a brief orthoptic examination to exclude ophthalmological disorders. No child with significant refractive error, squint, or ocular pathology is included in this report. Then the Dunlop test was administered 10 times. The procedure was similar to that followed in our first study, ${ }^{3}$ and also to that followed by Newman et al. ${ }^{4}$

In the test the child viewed two almost identical macular sized fusion scenes through a synoptophore. The slide viewed by the right eye had a house with a small pole to the left of the front door, while the left eye saw a house with a large pole to the right of the door. The angle of the synoptophore tubes was 
Dunlop responses and age

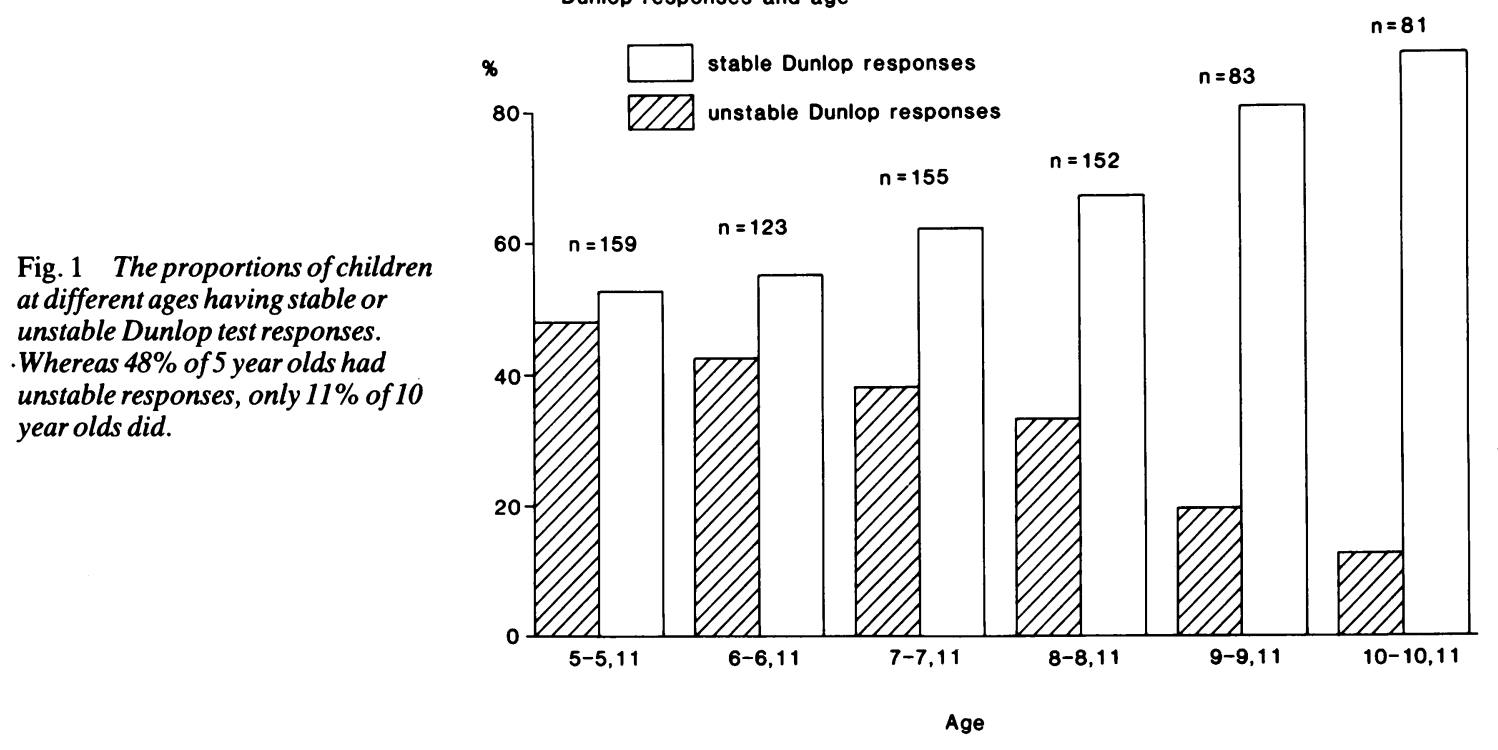

Fig. 1 The proportions of children at different ages having stable or unstable Dunlop test responses. -Whereas $48 \%$ of 5 year olds had unstable responses, only $11 \%$ of 10 year olds did.

adjusted until the two scenes fused. Then the orthoptist slowly abducted the synoptophore tubes (at $1-2 \%$, and the subjects attempted to diverge their eyes to maintain fusion. When children understand clearly what they have to do, most gain a clear impression that one of the poles seems to move towards the door during this procedure. After about $5^{\circ}$ divergence $\left(2 \frac{1}{1} 2^{\circ}\right.$ for each eye $)$ diplopia intervenes. On repeating the test 10 times, the slides being changed over frequently to try to prevent guessing, most children report movement of the pole on the same side on every trial. However, some children give inconsistent reports in the test. Their eyes did not diverge adequately, one or other of the poles seeming to disappear temporarily, and diplopia occurred much earlier after only $1^{\circ}$ or $2^{\circ}$ abduction of the tubes. The children reported that both the poles appeared to move either simultaneously or on successive occasions. If a child reported that both poles appeared to move during the test either simultaneously or successively on three or more trials, he/she was said to have unstable Dunlop test responses, a result denoting poor vergence control under these conditions.

For 451 of the children we could obtain reading ages from their teachers (Shonell single word, Neale or NFER group tests) together with some intelligence quotient (IQ) data. Although combining the results of different reading tests is undesirable, it is not likely to have caused systematic errors. We had individual IQ scores for only a few children, but all the children whose reading results we have included had IQs above 90 by class-administered tests.

\section{Results}

Of our total sample of 753 children aged 5-11 years $263(35 \%)$ were found to have unstable responses in the Dunlop test. But the proportion of children with stable responses increased year by year. Fig. 1 shows that, whereas only $52 \%$ of 5-6 year olds had gained stable responses, nearly $90 \%$ of $10-11$ year olds had done so. Thus among 7-11 year old children taken as a group we found that $27 \%$ showed unstable vergence control in the Dunlop test. Newman et al. found $56 \%$ of $7-11$ year olds to have abnormal Dunlop responses. ${ }^{4}$

We found that those of the 6-11 year old children who had achieved accurate vergence control were on average 6.3 months better at reading than their peers with unstable control. Reading ages for the 5 year olds were not available. The difference between stable and unstable groups was highly significant statistically $(\mathrm{p}<0 \cdot 01)$. A similar trend may be seen in some of Newman et al.'s results, but the statistical significance of this difference was not calculated.

In Fig. 2 the proportion of 6-11 year olds with stable or unstable Dunlop test responses is plotted as a function of quartile differences between reading and chronological age in the same way as Newman et al. presented their results. ${ }^{4} 44 \%$ of those whose reading was more than 18 months behind their chronological age (backward readers) had unstable responses in the Dunlop test, as did $40 \%$ of low normal readers, compared with $26 \%$ of high normal and only $14 \%$ of advanced readers (reading age more than 18 months ahead of chronological age). The 
Dunlop Test and reading

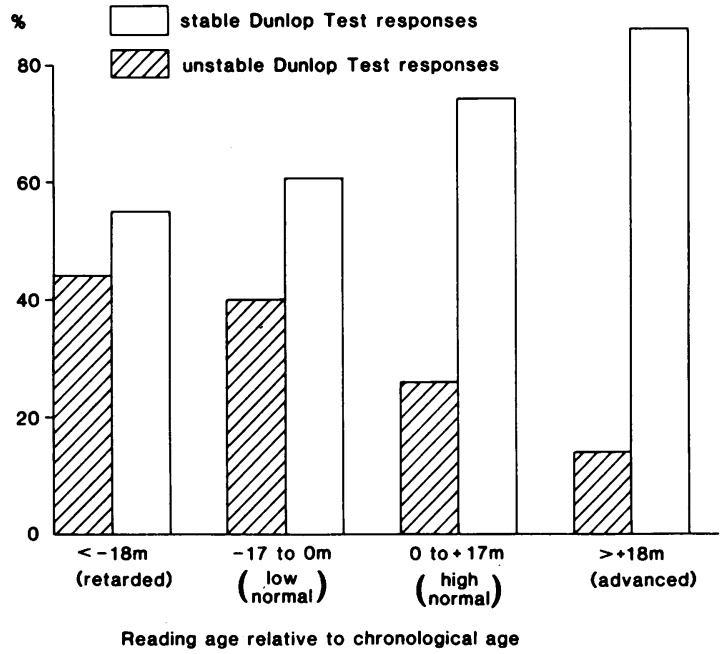

Fig. 2 The proportions of children of different reading levels relative to their age having stable or unstable Dunlop test responses.

differences between the groups were highly signifcant $\left(\chi^{2}=14.98 ; \mathrm{df}=3 ; \mathrm{p}<0.005\right)$. The same trends may be seen in Newman et al.'s results, but their differences were not statistically significant. ${ }^{4}$

\section{Discussion}

These results support the conclusions of our earlier study ${ }^{3}$ that the Dunlop test is useful in the investigation of children with reading disorders. In the sample reported here nearly half the children whose reading was more than 18 months behind their chronological age were found to have unstable responses in the Dunlop test compared with only $14 \%$ of advanced readers and $24 \%$ of all the children whose reading was ahead of their age. These differences were highly significant. Likewise the reading of those with unstable Dunlop test responses was on average over six months behind that of their peers with stable control.

Nevertheless in this study we found many more 'normal' readers with unstable vergence control than we did in 1982. There are a number of reasons for this. First, we chose exceptionally good readers as our controls in our earlier study. Their average reading age was over two years ahead of their chronological age. The figures reported here imply that less than $10 \%$ of such advanced readers would be expected to have abnormal Dunlop test responses. In addition the backward readers in our earlier study were much worse than most of the children reported here. Their reading was two to three years behind their chronological age; our present figures suggest that over $60 \%$ of such highly retarded readers would have abnormal Dunlop test responses.

Another way in which the results reported here differ from those of our previous study is that then the reading age of all the 160 children was measured by a single test, the Neale analysis of reading accuracy. However, we did not have the resources to assess the reading ages of all the children reported in the present study ourselves. Different reading tests were employed by the teachers of different groups of children. This is bound to have reduced the accuracy of the reading assessments. Adding 'noise' in this way, however, is likely to have reduced differences between the groups rather than increased them. Moreover it is unlikely to explain the systematic differences which we observed in the reading ability of children with stable as compared with those with unstable Dunlop test responses.

A most important additional reason why we observed more normal readers with abnormal Dunlop test responses among these unselected primary school children than in our earlier study is because we examined older children then. The average age of the children in our 1982 study was 10 years 1 month, whereas in our present sample (excluding the 5 year olds, for whom no reading ages were available) it was just over 7 years. We show here (Fig. 1) that the proportion of abnormal responses decreases with the age of the children. Whereas only $10 \%$ of 10 year olds had unstable responses on the Dunlop test, overall $30 \%$ of our sample of $6-11$ year olds showed poor vergence control. But the reading of the majority of children with unstable responses was low normal rather than seriously retarded. About a third of the 6-11 year old children we studied had unstable visuomotor control, yet the reading of most of these was within normal limits. This suggests that, although the normal development of visuomotor control was somewhat delayed in these children, most of them were not irreversibly affected and would probably catch up. But there is a residue of $5-10 \%$ of children whose acquisition of stable vergence was so delayed as possibly to lead to significant problems.

In our earlier study we suggested that children who had not achieved stable vergence control by the age of 10 may account for between half and two-thirds of children with specific reading retardation, or dyslexia. However, because we had inadequate IQ information in this study, we have not been able to relate reading to $I Q$, only to chronological age. Hence we cannot with any certainty identify any of the children in this study as truly dyslexic. If we had been able to express the children's reading as a 
function of their IQ more of those with abnormal Dunlop test responses might have been found to be poor readers relative to their IQ, and more children with normal responses but lower IQ might have been classified as advanced readers in relation to their IQ.

Although the same trends as we report here may be seen in some of the results of Newman et al., they found many more normal and advanced readers with abnormal Dunlop test responses ${ }^{4}$ than we did. So the differences between their groups were not statistically significant. Newman et al. suggested that in our first study we may have been biased by knowing that our clinic referrals were likely to have been mainly reading retarded while our primary school children were mostly normal readers. However, in the present study we examined an unselected group of children in their schools without even such general prior knowledge of their reading, yet found that those with abnormal Dunlop test responses were very significantly overrepresented among the poorer readers.

We have only just begun to appreciate the unreliability of this test in inexperienced hands. It relies heavily on the ability of young children to concentrate on an unfamiliar and complex perceptuomotor task. Accordingly the person administering it needs to be highly experienced with working with children and interpreting their reactions, to make sure that they have really understood the task and are not merely trying to guess as a result of their natural desire to please. We have recently shown ${ }^{8}$ that we can assess a child's vergence control objectively by recording the movements of each eye during the Dunlop or other synoptophore vergence tests. Such recordings provide a more reliable indicator of visuomotor abnormalities in reading by retarded children. They are also more satisfactory for monitoring procedures aimed at improving vergence control, such as monocular occlusion. ${ }^{\prime}$

It has been suggested that low normal and retarded readers have unstable responses in the Dunlop test merely because they find it difficult to understand the test. However, the instructions for the Dunlop test are verbal; they do not involve any reading. Moreover we have shown that whether children have unstable responses in the Dunlop test ${ }^{3}$ or whether they respond to monocular occlusion treatment by developing stable visuomotor control' is independent of their IQ. Many highly intelligent dyslexics have unstable Dunlop test responses, whereas many children with below average IQs can understand the instructions in the Dunlop test sufficiently well to give stable responses.

It has also been suggested that rather than unstable visuomotor control causing reading difficulties cause and effect are the other way round-that is, children develop reliable vergence control after they have learnt to read. However, we have shown that a reading spurt, if it occurs in dyslexics, always follows acquisition of stable Dunlop test responses and usually cannot proceed without such development of reliable visuomotor control.' Thus it seems likely that developing accurate vergence control is a vital prerequisite for learning to read normally. This explains why at all ages those with unstable Dunlop test responses tend to be worse readers than their peers with stable vergence control.

We thank Mr D Brownhill, chief psychologist, Berks Education Department, for his help and support and the Oxford Regional Health Authority for financial assistance.

\section{References}

1 Stein J, Fowler, S. Monocular occlusion may improve visuomotor perception and reading in some dyslexic children. Lancet 1985; ii: 69-72.

2 Dunlop P. Dyslexia: the orthoptic approach. Aust Orthopt J 1972; 12: 16-20.

3 Stein J, Fowler MS. Diagnosis of dyslexia by means of a new indicator of eye dominance. BrJ Ophthalmol 1982; 66: 332-6.

4 Newman SP, Wadsworth JF, Archer R, Hockly R. Ocular dominance, reading, and spelling ability in school children. $\mathrm{Br} \mathrm{J}$ Ophthalmol 1985; 69: 228-32.

5 Stein JF, Fowler MS. Ocular motor dyslexia. Dyslexia Rev 1982; 5: $25-8$.

6 Stein JF, Fowler MS. A physiological theory of visual dyslexia. In: Rose FC, ed. Advances in neurology: Progress in aphasiology. New York: Raven Press, 1984: 42.

7 Stein JF, Fowler MS. Ocular motor problems of learning to read. In: Gale AG, Johnson F, eds. Theoretical and applied aspects of eye movement research. Amsterdam: Elsevier, 1984.

8 Stein JF, Riddell PM, Fowler MS. Vergence eye movements in normal and dyslexic children. J Physiol (Lond) 1985; 369: 41.

Accepted for publication 29 August 1985. 